\title{
Effectiveness of yoga asanas over conventional physiotherapy treatment on functional outcomes in patients with knee osteoarthritis
}

\begin{abstract}
Background: The purpose of this study was to check the effectiveness of 4 weeks of yoga asanas exercises on patients with OA knee in comparison with physiotherapy interventions.

Methods: Fifty-Eight subjects between 40-55 years of age diagnosed with Osteoarthritis of Knee (OA Knee) was randomly allocated into two groups. Group A received Yoga Program for 4 weeks and Group B received Conventional Physiotherapy for 4 weeks. The outcomes assessed in terms of Visual Analog Scale (VAS), WOMAC, 30Second Chair Stand Test and the knee range of Motion (ROM) at baseline, at end of week 2 , and at the end of week 4

Results: Findings showed that all the outcome measures were significantly improved in both the groups. However, there was no significant clinical difference in betweengroup comparisons at the end of week 2 , and at the end of week 4.

Conclusion: This study concludes that the yoga program is more effective in improving the functionality and mobility outcome measures of the OA knee patients than a conventional physiotherapy program.
\end{abstract}

Keywords: sosteoarthritis of knee, conventional physiotherapy, yoga asanas, 30second chair stand test, womac
Volume 3 Issue 2 - 2018

\author{
Patel Krishnakumari Rakeshbhai,' \\ Bid Dibyendunarayan Dhrubaprasad,' \\ Thangamani Ramalingam Alagappan,' \\ Shahanawaz SD ${ }^{2}$ \\ 'Department of Musculoskeletal Physiotherapy, Sarvajanik \\ College of Physiotherapy, Surat, India \\ ${ }^{2}$ Department of Neurophysiotherapy, MGM Institute of \\ Physiotherapy, Aurangabad, India
}

\section{Correspondence: Department of Musculoskeletal Physiotherapy, Sarvajanik College of Physiotherapy, Surat, India +9194271397| IEmail dnbid7 I@gmail.com}

Received: May 09, 2018 | Published: May 17, 2018

\section{Introduction}

Osteoarthritis is a condition described as an articular cartilage degeneration and a subsequent periarticular bone response. ${ }^{1}$ The global prevalence rate of OA is $29 \%$ for men, $47 \%$ for women. ${ }^{2} \mathrm{OA}$ is a group of overlapping but different diseases which may have a diverse etiology, but with similar biology, morphology, and clinical outcomes. The disease processes not only affect the articular cartilage, and but also involves the entire joint, including the subchondral bone, ligament, capsule, synovial membrane, and periarticular muscles in general. Eventually, the articular cartilage degenerates with fibrillation, fissures, and full thickness loss of joint surface. ${ }^{3}$ The pathology of OA encompasses the entire joint in a disease process that involves focal and progressive hyaline articular cartilage loss with simultaneous changes in the bone beneath the cartilage, including the development of marginal osteophytes, and increased bony sclerosis. Soft-tissue structures in and around the joint are also affected. These structures include synovium, which may show some inflammatory infiltrates; ligaments, which are often lax; and muscles across the joint, which becomes weak. There are many people with radiographic and pathologic proof of OA knee have no symptoms. From a clinical viewpoint, the most persuasive definition of disease can be given as 'one that combines the pathology of disease with pain that happens with joint use'. However, the exact cause of pain in osteoarthritis is remains unknown. ${ }^{4}$ It is essential to separate the disease process of $\mathrm{OA}$ and the set of symptoms of musculoskeletal pain and disability apart; because the two are only inadequately correlated. Though OA is related with increasing age, obesity, previous deformity, injury, and ligamentous laxity, but the broader clinical presentation of musculoskeletal pain and disability is predicted by increasing age, osteoarthritis severity, obesity, lack of exercise, low personal self- efficacy, and co-occurring conditions caused by alcohol, smoking, and some other risk factors such as depression, poor socioeconomic status and low educational level etc. ${ }^{4}$ Treatment methods of osteoarthritis of knee can be allocated into three distinct groups: Medical, Surgical, and Allied medical treatment. Under medical treatment, non-steroid anti-inflammatory medicines and intraarticular injection of corticosteroids are used. These medicines have numerous harmful side effects on cardiovascular system, digestive system, and kidneys. ${ }^{5,6}$ In surgical treatment debridement and osteotomies are done, which gives only temporary reliefs and may delay the condition's progress. As a last option total or unicompartmental knee arthroplasty is used. Sometimes in rare cases knee joint arthrodesis is also used. Though Knee Arthroplasty is a generally recognized treatment is severe osteoarthritis of knee. But it is very stressful for the patient and the family. It is an expensive and time-consuming process for generally old patients and they find it tough to tolerate the surgical procedures. ${ }^{7}$ Allied medical treatments for osteoarthritis of knee include physiotherapy, acupuncture, use of assistive devices like a crutch, knee brace, insoles and heel wedges, losing weight, and exercise. ${ }^{7-10}$ Yoga is considered a mind-body practice in alternative or complementary medicine. It integrates various body poses, breathing techniques, and meditation; which can theoretically decrease pain and stiffness related to OA by realigning the skeletal structures, strengthening muscles around the joints, and stretching tight joint structures. The frequent joint motion when practicing yoga, is thought to have some physiologic effects at the tissue or cellular level. Yoga exercises may reduce fluid pressure, which in turn, preserves cartilage that would have been lost by immobilization. It is observed that yoga exercises improves balance, flexibility, strength, and relaxation in the general adult population, and which has physiological benefits 
comparable to those of moderate exercise. Yoga is presently one of the fitness programs recommended by the Arthritis Foundation (AF) to encourage joint flexibility and lower stress to possibly benefit patients with osteoarthritis in common. ${ }^{11}$ Management of OA knee usually targeted on symptom control and in the first instance, conservative allied medical management (e.g. exercise, weight loss) is promoted by most treatment guidelines. Physiotherapy treatment intends to relieve pain, improve function, mobility, knee stabilization, promote adaptation of certain activities of daily life, reduce the load on the joint and prevent deformities; may slow down the progression of the disease and improve the quality of life of affected population. ${ }^{9}$ Conducted a review of systematic reviews related to physiotherapy for OA Knee and found that only exercise and weight reduction provided high-quality evidence for improvement in pain and function. Acupuncture, Low-Level Laser Therapy (LLLT) and Transcutaneous Electrical Nerve Stimulation (TENS) could provide the moderate quality of evidence for the same pain and function variables. While other interventions accomplished a low quality evidence or no- evidence. ${ }^{12}$ The present study was conducted to compare the effectiveness of 'Yoga Asanas' over 'Conventional Physiotherapy' treatment on functional outcomes [30-second chair stand test, Visual analog scale, Knee Range Of Motion, and The Western Ontario McMaster Universities Osteoarthritis Index (WOMAC)] in patients with OA knee.

\section{Methods}

This study used a quasi-experimental design and there were no dropouts in the study. Inclusion Criteria: Patients of both genders of 40-55 years of age having Grade- 2 or Grade- 3 unilateral OA of the knee (Kellgren-Lawrence Classification of Osteoarthritis) and having average pain rating from 3 to 7 on a numerical pain rating scale. Exclusion criteria: Those who had a history of any knee surgery in last six month, or history of knee injection in the last four week, or having any inflammatory arthritis and BMI $>30 \mathrm{~kg} / \mathrm{m}^{2}$ were excluded. The ethical approval for this study was taken from the Ethics Committee of 'The Sarvajanik College of Physiotherapy', Surat. Participants were given explanation about the purpose of the study and if they agreed then a written informed consent was obtained. All the participants underwent a baseline assessment prior to treatment using 30-second chair stand test, Knee Range of Motion, Visual analog scale, and WOMAC

Group A Yoga Program performed following Yoga-Asanas for 30 minutes for each session, 3 times per week for 4 weeks. Yoga asanas included Tadasana, Vrksasana, Utkatasana, Pawanmuktasana,
Virabhadrasana, Janu Sirsasana, and Utthita Trikonasana. ${ }^{13,14}$ The procedure for doing these yoga asanas can be obtained from many books on yoga asanas. ${ }^{13,14}$ Each exercise were performed for a total of 3 sets. Each exercise was consist of total 10 repetitions, with short intervals of rest in between.

Group-B Conventional Physiotherapy Program was done for 30minutes each session, 3 times per week for 4 weeks. This program included Isometrics Quadriceps exercise ${ }^{15}$ Straight leg raising (SLR) exercise, Terminal knee extension or VMO strengthening exercise, active knee flexion \& extension in supine and interferential current (two pole method, and beat frequency $100 \mathrm{~Hz}$ ). Each exercise was performed for a total of 3 sets. Each set was made up of 10 repetitions.

\section{Outcome measures}

Following outcome measures were used: (a) 30second chair stand test- this test measures the maximum number of the chair stand repetitions possible in a 30 second period. The ideal height chair is used for testing. The starting position of subjects was standardized including buttock placement, back support, use of hands and foot placement. Arms were crossed at the wrists and held against the chest. The patients were asked to sit and stand quickly and safely as possible for 30 seconds. ${ }^{16}$ (b) Visual analog scale-The pain VAS is a one-dimensional measure of pain intensity. For pain intensity, the scale is most commonly anchored by "no pain" (score of 0 ) and "pain as bad as it could be" or "worst imaginable pain (score of 10). ${ }^{17}$ (c) The range of motion- Knee flexion and extension ROM in degrees were measured bilaterally in a supine position. ${ }^{18}$ (d) The Western Ontario McMaster Universities Osteoarthritis Index (WOMAC)-A disease-specific index of disability, was used as a subjective measure of perceived health and physical function.

\section{Statistical analysis}

Normality of data was checked using Shapiro-Wilk test. As the data were not normally distributed, hence nonparametric test was used. The data have been presented as Mean and SD or Median and Interquartile range (IQR) for all the outcome measures as the outcome measures were measured at multiple time (Day 1, Post 2 Week and Post 4 Week) intervals. Friedman ANOVA test was used as primary statistical analysis for within group comparison. For between groups comparisons each pre and post period were investigated by Mann Whitney $U$ test. Statistical significance was set at $\mathrm{P}<0.05$ for all statistical analyses. All data analyses were performed by using the SPSS version 20.0 (IBM, Armonk, NY, USA) for Windows Figure 1.

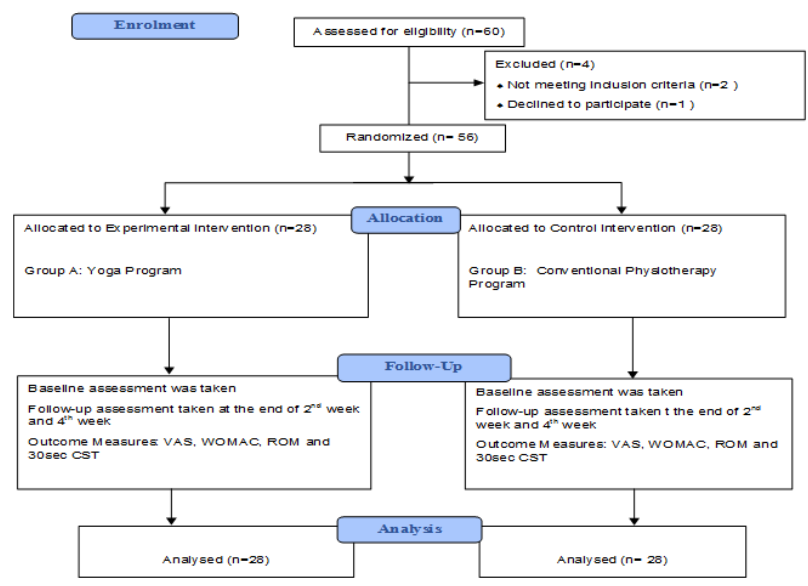

Figure I Flow diagram of the study. 


\section{Results}

The present study included 56-patients with osteoarthritis of knee, who were randomly allocated to Group-A: (Yoga Program) with Age: $45.36 \pm 4.916$ Years, Male + Female: $8+20$, height: $166.68 \pm 7.1 \mathrm{~cm}$, weight: $63.46 \pm 12.09 \mathrm{~kg}$, BMI: $19.17 \pm 3.6)$ and Group-B: (Conventional Physiotherapy Program) with Age: 51.11 \pm 4.57 Years, Male/Female: $8 / 20$, height: $164.32 \pm 9.4 \mathrm{~cm}$, weight: $61.54 \pm 8.78 \mathrm{~kg}$, BMI: 18.77 \pm 2.85 ). The present study investigates the comparative effectiveness of 'Yoga Asanas' over 'Conventional Physiotherapy' treatment on functional outcomes such as $30-$ second chair stand test,
Visual analog scale, Knee Range of Motion, and WOMAC in patients with OA knee. The descriptive statistics have been presented as Mean and SD or Median and IQR for all the outcome measures which are measured at multiple time (Day 1, Post 2 Week and Post 4 Week) intervals. Friedman ANOVA test for within group (repeated measures) comparison showed a significant difference in score for all the outcomes to both the groups. The Mann Whitney U test done at end of 4 weeks after the interventions for group comparison showed that the yoga group was significantly different in functionality (WOMAC) and mobility (CST) outcomes than the conventional group Table 1.

Table I Between and within group comparisons for the outcome measures

\begin{tabular}{|c|c|c|c|c|c|c|}
\hline \multirow{2}{*}{$\begin{array}{l}\text { Outcome } \\
\text { Measures }\end{array}$} & \multirow{2}{*}{$\begin{array}{l}\text { Measurement } \\
\text { Levels }\end{array}$} & \multicolumn{2}{|c|}{ Group-A: (Yoga Program) } & \multicolumn{2}{|c|}{$\begin{array}{l}\text { Group-B: (Conventional } \\
\text { Physiotherapy Program) }\end{array}$} & \multirow{2}{*}{ P-Value } \\
\hline & & $\operatorname{Mean} \pm$ SD & Median (IQR) & Mean \pm SD & Median (IQR) & \\
\hline \multirow{4}{*}{ VAS } & Baseline & $61.25 \pm 5.7$ & $60.5(57-65)$ & $61.43 \pm 5.4$ & $61(57-66)$ & 0.902 \\
\hline & Post 2-Weeks & $54.14 \pm 5.0$ & $53.5(50-58)$ & $54.36 \pm 5.4$ & $53(50-59)$ & 0.882 \\
\hline & Post 4-Weeks & $49.11 \pm 4.2$ & $49(46-52)$ & $47.82 \pm 5.3$ & $47(43-53)$ & 0.3 \\
\hline & P Value & 0.001 & & 0.001 & & \\
\hline \multirow{4}{*}{ WOMAC } & Baseline & $28.50 \pm 7.3$ & $27.08(24.4-34.1)$ & $29.17 \pm 5.3$ & $29.16(26.0-31.7)$ & 0.549 \\
\hline & Post 2-Weeks & $16.75 \pm 4.4$ & $16.14(13.5-20.3)$ & $18.86 \pm 3.9$ & $17.7(16.6-19.0)$ & 0.065 \\
\hline & Post 4-Weeks & $10.60 \pm 2.6$ & $10.41(9.3-12.05)$ & $12.83 \pm 2.7$ & 11.97(10.6-11.9) & $0.002 *$ \\
\hline & P Value & 0.001 & & 0.001 & & \\
\hline \multirow{4}{*}{ CST } & Baseline & $11.18 \pm 1.2$ & $11(10-12)$ & $11.32 \pm 1.8$ & $12(9-12)$ & 0.783 \\
\hline & Post 2-Weeks & $13.39 \pm 1.4$ & $13(13-14)$ & $13.36 \pm 1.9$ & $14(12-15)$ & 0.796 \\
\hline & Post 4-Weeks & $14.18 \pm 0.9$ & $14(14-14.75)$ & $15.29 \pm 2.1$ & $16(13-17)$ & $0.013 *$ \\
\hline & P Value & 0.001 & & 0.001 & & \\
\hline
\end{tabular}

\section{Discussion}

The present study showed that both the treatment group achieved statistically meaningful improvement in pain (VAS), functionality (WOMAC) and mobility (30Second Chair Stand Test) for within group comparisons but clinically there was no difference in between-group comparisons. There was no change in knee ROM in both the groups at the end of $4^{\text {th }}$ week of the study. ${ }^{19}$ found that 8 weeks of Hatha yoga exercises on women with OA knee decreased the pain scores in yoga group compared to conventional physiotherapy group. In our study there was a statistically significant improvement in pain score was seen for Yoga Group compared to conventional Physiotherapy Group. But that was not enough to be a clinically important difference. As we know that a mean reduction in VAS of $30.0 \mathrm{~mm}$ represents a clinically important difference in pain severity that matches with patients' perception of adequate pain control. Sharon L. ${ }^{20}$ reported statistically significant reductions in WOMAC Pain, WOMAC Physical Function, in OA knee patients using yoga interventions. But in the present study, there was no clinically significant difference found at post 2 weeks and 4 weeks of intervention for the between-group comparison of WOMAC outcome, because it is understood that in therapeutic intervention, the effects larger than $12 \%$ of baseline score $(6 \%$ of maximal score) can be detected as MCID for the WOMAC. ${ }^{21,22}$ found significant improvement in the score of 30second chair stand test using Hatha yoga exercise program in managing symptoms related to OA knee in aged women. ${ }^{23}$ Also showed an improvement in mobility of patient after the yoga intervention. Our study also showed a significant improvement in scores of for $30 \mathrm{sec}$ chair stand test for yoga group..$^{24}$ showed a greater decrease in pain, anxiety, and morning stiffness in the patients with OA knee in the yoga group. Similarly, our study has also shown improvement in the yoga group..$^{25}$ found a substantial improvement in pain, health, strength, and flexibility in patients with knee OA using hath yoga in sedentary people. suggested that yoga may provide a possible treatment choice for previously nonpractitioner of yoga, obese patients with $>50$ years of age and suggests possible reductions in pain and disability caused by OA knee. ${ }^{26}$ also found better results with yoga group for functional outcome measures compared with conventional treatment in OA patients. But in our study, we did not find any significant clinical difference in betweengroup comparisons in patients with knee OA. In our study, limitations were: (a) data collection by an independent observer was not done and (b) there was no placebo control group in the study.

\section{Conclusion}

This study concluded that the yoga program is more effective in improving the functionality and mobility outcome measures of the $\mathrm{OA}$ knee patients than a conventional physiotherapy program. Considering the similar effects of conventional physiotherapy programs on most of the outcome measures, yoga could well be added to that to gain the 
additive effect in the treatment of OA knee patients. Moreover, yoga is a client-driven practice; it may be recommended as a home program along with other therapeutic measures.

\section{Acknowledgements}

None.

\section{Conflict of interest}

The authors declared that no conflict of interest exists

\section{References}

1. Felson DT, Lawrence RC, Dieppe PA, et al. Osteoarthritis: new insights. Part 1: the disease and its risk factors. Annals of internal medicine. 2000;133(8):635-646.

2. Lawrence JS, Bremner JM, Bier F. Osteo-arthrosis. Prevalence in the population and relationship between symptoms and $\mathrm{x}$-ray changes. Annals of the rheumatic diseases. 1966;25(1):1-24.

3. Cole AA, Kuettner KE. MMP-8 (neutrophil collagenase) mRNA and aggrecanase cleavage products are present in normal and osteoarthritic human articular cartilage. Acta Orthopaedica Scandinavica.1995;66(266):98-102.

4. Felson DT, Lawrence RC, Dieppe PA, et al. Osteoarthritis: New insights. part 1: the disease and its risk factors. Annals of Internal Medicine. 2000;133(8):635-646.

5. Thomas A, Eichenberger G, Kempton C, et al. Recommendations for the treatment of knee osteoarthritis, using various therapy techniques, based on categorizations of a literature review. Journal of geriatric physical therapy. 2009;32(1):33-38

6. Kawasaki T, Kurosawa H, Ikeda $\mathrm{H}$, et al. Therapeutic home exercise versus intraarticular hyaluronate injection for osteoarthritis of the knee: 6-month prospective randomized open-labeled trial. Journal of the Japanese Orthopaedic Association. 2009;14(2):182-191.

7. Lin CW, Taylor D, Bierma-Zeinstra SM, et al. Exercise for osteoarthritis of the knee. Physical therapy. 2010;90(6):839-842.

8. Egan BA, Mentes JC. Benefits of physical activity for knee osteoarthritis: a brief review. Journal of gerontological nursing. 2010;36(9):9-14.

9. Jamtvedt G, Dahm KT, Christie A, et al. Physical therapy interventions for patients with osteoarthritis of the knee: an overview of systematic reviews. Physical therapy. 2008;88(1):123-136.

10. Bukowski EL, Conway A, Glentz LA, et al. The effect of iyengar yoga and strengthening exercises for people living with osteoarthritis of the knee: a case series. International quarterly of community health education. 2006;26(3):287-305.

11. Cheung C, Wyman JF, Resnick B, et al. Yoga for managing knee osteoarthritis in older women: a pilot randomized controlled trial. $B M C$ Complementary and Alternative Medicine. 2014;(14):1-160.

12. Ferreira de Meneses SR, Hunter DJ, Young Docko E, et al. Effect of low-level laser therapy $(904 \mathrm{~nm})$ and static stretching in patients with knee osteoarthritis: a protocol of randomised controlled trial. $B M C$ Musculoskelet Disord. 2015;(16):1-252.
13. Svatmarama S. The Hatha Yoga Pradipika. New York; 2002.

14. Saraswati SS. Asana Pranayama Mudra Bandha: Yoga Publications Trust. India. 1996

15. Anwer S, Alghadir A. Effect of Isometric Quadriceps Exercise on Muscle Strength, Pain, and Function in Patients with Knee Osteoarthritis: A Randomized Controlled Study. Journal of Physical Therapy Science. 2014;26(5):745-748.

16. Unver B, Kalkan S, Yuksel E, et al. Reliability of the 50-foot walk test and 30-sec chair stand test in total knee arthroplasty. Acta Ortopedica Brasileira. 2015;23(4):184-187.

17. Hawker GA, Mian S, Kendzerska T, et al. Measures of adult pain: Visual Analog Scale for Pain (VAS Pain), Numeric Rating Scale for Pain (NRS Pain), Mc Gill Pain Questionnaire (MPQ), Short-Form McGill Pain Questionnaire (SF-MPQ), Chronic Pain Grade Scale (CPGS), Short Form-36 Bodily Pain Scale (SF-36 BPS), and Measure of Intermittent and Constant Osteoarthritis Pain (ICOAP). Arthritis care \& research. 2011;63 (11):S240-S252.

18. Mascarin NC, Vancini RL, Andrade MldS, et al. Effects of kinesiotherapy, ultrasound and electrotherapy in management of bilateral knee osteoarthritis: prospective clinical trial. BMC Musculoskeletal Disorders. 2012;13(1):1-182.

19. Ghasemi GA, Golkar A, Marandi SM. Effects of Hata Yoga on Knee Osteoarthritis. International Journal of Preventive Medicine. 2013;4(1):S133-S138.

20. Kolasinski SL, Garfinkel M, Tsai AG, et al. Iyengar yoga for treating symptoms of osteoarthritis of the knees: a pilot study. Journal of alternative and complementary medicine. 2005;11(4):689-693.

21. Angst F, Aeschlimann A, Stucki G. Smallest detectable and minimal clinically important differences of rehabilitation intervention with their implications for required sample sizes using WOMAC and SF-36 quality of life measurement instruments in patients with osteoarthritis of the lower extremities. Arthritis and rheumatism. 2001;45(4):384-391.

22. Cheung C, Wyman JF, Bronas U, et al. Managing knee osteoarthritis with yoga or aerobic/strengthening exercise programs in older adults: a pilot randomized controlled trial. Rheumatology international. 2017;37(3):389-398.

23. Brenneman EC, Kuntz AB, Wiebenga EG, et al. A Yoga Strengthening Program Designed to Minimize the Knee Adduction Moment for Women with Knee Osteoarthritis: A Proof-of-Principle Cohort Study. PloS one. 2015;10(9):e0136854.

24. Ebnezar J, Nagarathna R, Yogitha B, et al. Effect of integrated yoga therapy on pain, morning stiffness and anxiety in osteoarthritis of the knee joint: A randomized control study. International Journal of Yoga. 2012;5(1):28-36.

25. Moonaz SH, Bingham CO, Wissow L, et al. Yoga in Sedentary Adults with Arthritis: Effects of a Randomized Controlled Pragmatic Trial. The Journal of rheumatology. 2015;42(7):1194-1202.

26. Awasthi V, Singh A, Verma N, et al. Effect of Yoga practices on clinical profile of Osteoarthritis Knee: A randomized control trial. International Journal of Yoga and Allied Sciences. 2013;2(2):91-96. 